\title{
Formación de la identidad profesional frente a la crisis global socioambiental
}

\section{Professional Identity Formation in a Global Socio-Environmental Crisis}

\author{
David Cuadra-Martínez ${ }^{\mathrm{a}, \mathrm{b}, *}$, Pablo J. Castro-Carrascoc ${ }^{\mathrm{c}}$, Cristian Oyanadel ${ }^{\mathrm{d}}$, \\ Ingrid González ${ }^{c}$, José Sandoval-Díaz ${ }^{\mathrm{e}}$, Daniel Pérez-Zapata ${ }^{\mathrm{f}}$ \\ ${ }^{a}$ Facultad de Humanidades y Educación, Universidad de Atacama, Chile \\ bFacultad de Psicología, Universidad del Aconcagua, Argentina \\ 'Facultad de Humanidades, Universidad de La Serena, Chile \\ dDepartamento de Psicología, Universidad de Concepción, Chile \\ eDepartamento de Psicología, Universidad del Bio Bio, Chile

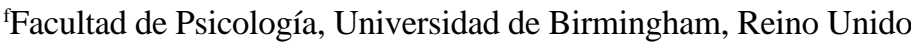

\section{Resumen}

Antecedentes: nos encontramos ante una crisis global que amenaza la subsistencia del planeta. Aunque durante las últimas décadas se han propuesto un conjunto de medidas para enfrentar esta crisis global, los resultados no han sido auspiciosos. Estado del arte: dado el importante rol de los profesionales en la sociedad, en este trabajo se argumenta que un desarrollo sistemático de la identidad profesional en la formación inicial es clave para el desarrollo sustentable. Se propone que esta identidad profesional podría construirse en base a las dimensiones (a) integración de saberes subjetivos, profesionales y científicos, (b) creatividad para abordar los problemas actuales y futuros, (c) prosocial y (d) ecológica. El objetivo de este trabajo es sustentar la urgencia de lo mencionado para dar respuesta a la crisis global y proporcionar sugerencias que orienten el desarrollo sistemático de la identidad profesional durante la formación inicial, en base a estas dimensiones. Conclusiones: a partir de la literatura científica, se argumenta la necesidad de formar sistemáticamente la identidad profesional en base a estas dimensiones, proporcionando sugerencias al respecto.

Palabras clave: crisis global; identidad profesional; prosocial; ecológico; proambiental; creatividad.

Para citar este artículo:

Cuadra-Martínez, D., Castro-Carrasco, P. J., Oyanadel, C., González, I., Sandoval-Díaz, J., \& Pérez-Zapata, D. (2021). Formación de la identidad profesional frente a la crisis global socioambiental. Liberabit, 27(2), e504. https://doi.org/10.24265/liberabit.2021. v27n2.05

* david.cuadra@uda.cl

\begin{abstract}
Background: We are facing a global crisis that threatens the survival of the planet. Although during the last decades a set of measures have been suggested to tackle this global crisis, results have not been auspicious. State of the art: Given the important role of professionals in modern societies, in this work it is argued that a systematic development of professional identity during the initial training is key for sustainable development. It is proposed that this professional identity could be built based on the following dimensions: (a) integration of subjective, professional, and scientific knowledge, (b) creativity to address current and future problems, (c) prosociality, and (d) ecology. The objective of this work is to support such urgency in order to respond to the global crisis and provide suggestions that guide the systematic development of professional identity during the initial training based on these dimensions. Conclusions: From the review of scientific literature, the need to systematically form the professional identity based on the aforementioned dimensions is argued, and suggestions are provided in this regard.
\end{abstract}

Keywords: global crisis; professional identity; prosocial; ecological; pro-environmental; creativity.

Este es un artículo Open Access publicado bajo la licencia Creative Commons Atribución 4.0 Internacional. (CC-BY 4.0)

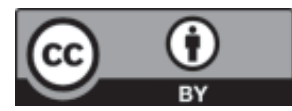

Universidad de San Martín de Porres, Lima - Perú http://ojs3.revistaliberabit.com 


\section{Introducción}

En mayo del año 2021, la Organización de Naciones Unidas (ONU) tituló la siguiente noticia «Los conflictos y las crisis económicas empujan a la inseguridad alimentaria a su nivel más alto en cinco años» (ONU, 2021a). En un escenario igual de aterrador, The New York Time dedica una página exclusiva para informar los desastres socionaturales que frecuentemente afectan a la humanidad, siendo Latinoamérica una de las regiones más dañada por el cambio climático (ONU, 2021b). Junto con esto, sin duda la experiencia más impactante que ha golpeado a la humanidad en los últimos cien años es la pandemia por el COVID-19 y la consecuente crisis global que ha amenazado la subsistencia y el desarrollo de las comunidades a nivel planetario.

A pesar del desarrollo científico, tecnológico y económico en el mundo en general, el futuro de la humanidad no parece tan prometedor. Bradshaw et al. (2021) advierten de una insuficiente y lenta respuesta frente al acelerado daño ambiental planetario, además de una falta de reconocimiento de los enormes desafíos para crear un futuro sustentable.

Nos encontramos en una crisis humanitaria multidimensional en rápido aumento (Glavic, 2020), con un incremento de la violencia para la solución de conflictos, la exacerbación de las desigualdades sociales y el aumento de la vulnerabilidad de los grupos más desfavorecidos, la migración forzada, el deterioro de la salud mental, la dificultad para la regulación de la carrera armamentista de los países, entre otras problemáticas (ONU, 2020).

Los desafíos actuales y futuros exigen replantear las formas de convivir en sociedad y de relacionarnos con el medioambiente. En este escenario, la educación representa una voz de esperanza para prevenir, mitigar y adaptarse ante la crisis socioecológica que el actual panorama cimienta (Glavic, 2020). Más que nunca necesitamos de una ciudadanía más prosocial y ecológica, y de profesionales preparados para enfrentar hábilmente los desafíos que impone la realidad (Ribers et al., 2021).

Sin embargo, aunque la educación ambiental se viene planteando desde hace décadas (Glavic, 2020), incluso la UNESCO (2014) se encuentra impulsando una agenda con la meta de reorientar y fortalecer la educación para el desarrollo sustentable, los resultados logrados a la fecha no son suficientes para revertir la crisis global.

Una potencial solución viable es la formación profesional universitaria. Los profesionales ocupan un lugar importante en la sociedad, porque son quienes se encargan de la educación formal, identifican problemas y prestan servicios para resolverlos (Fitzgerald, 2020). Desde un enfoque social del desarrollo de la identidad profesional (IP), a los profesionales se les identifica con la capacidad de adaptarse y constituirse como agentes de transformación social (Cardoso et al., 2014). Son indispensables para la economía cognitiva de la sociedad actual, que requiere de sistemas intelectuales, gestión del conocimiento y tecnologías para su desarrollo (Gorlacheva \& Tikhomirova, 2021).

No obstante, de la relevancia de los atributos con que la sociedad identifica a los profesionales, el desarrollo de la IP ha sido poco considerada en las políticas de educación superior y en la formación profesional (Moorhead, 2019). Orientar la formación profesional hacia la construcción de la IP implica un salto sustantivo desde el solo desarrollo de competencias para el desempeño laboral, hacia la construcción de un sentido profundo y arraigado de lo que significa ser profesional, y del rol profesional a desempeñar en una sociedad. Además, la identidad construida también puede guiar la forma en que los profesionales se relacionan medioambientalmente (Riggs, 2015).

Aunque aprender la profesión es construir en algún nivel la IP, se necesita de un proceso formativo sistemático para lograr la necesaria construcción 
consciente de lo que significa actualmente ser profesional en una sociedad en crisis. También es necesaria una integración reflexiva y consciente de las metas, necesidades, aspiraciones profesionales y demandas sociales para hacer frente a los graves problemas humanitarios. Junto con esto, es indispensable que la cada vez mayor especialización disciplinaria, conviva con la necesaria mirada global de los complejos sistemas adaptativos, para lo cual se necesita integrar nuevos saberes y capacidades como parte de esta identidad que posibiliten un pensamiento más complejo y transdisciplinar. Se torna urgente formar una IP fuertemente arraigada, creativa, solidaria, ecológica y cuestionadora del modelo económico neoclásico que identifica a las profesiones como un eslabón del mercado, bajo la lógica de un sistema de recursos inagotables y de crecimiento infinito, que inhibe la agencia, la creatividad y la crítica hacia el modelo imperante (Elizalde \& De la Cuadra, 2019).

En este trabajo se argumenta que un desarrollo sistemático y planificado de la IP en la formación inicial, es clave para el desarrollo sustentable. Se propone que esta IP debe construirse considerando la integración de saberes subjetivos, profesionales y científicos que posibiliten una adecuada creatividad y previsibilidad para abordar los problemas actuales y futuros, además de las dimensiones prosocial y ecológica. El objetivo, por lo tanto, es sustentar la gran importancia de abordar la crisis global desde la formación de la IP, para dar respuesta a esta grave problemática y proporcionar sugerencias que orienten el desarrollo sistemático de la IP durante la formación inicial, incluyendo las dimensiones mencionadas.

\section{Crisis global}

Aunque en el transcurso de la historia la humanidad ha estado expuesta a diversos peligros medioambientales, el panorama actual parece ser un problema estructural de tal magnitud, al punto de encontrarse en riesgo la subsistencia de las especies que habitan el planeta (Tregidga \& Laine, 2021).
Actualmente, el mundo enfrenta una crisis global definida en este trabajo como un periodo (el siglo XXI) en donde, ambientalmente, no se logra el debido equilibrio ecológico que permita un adecuado ciclo para la sustentabilidad de las especies debido a la acción antrópica (Dasgupta, 2021). Sumado a esto, en una dimensión social, las formas de convivir tampoco logran el adecuado equilibrio entre el desarrollo personal y el colectivo, entre la colaboración y la competitividad para el desarrollo, produciendo graves problemas psicosociales y ecológicos.

Para esto, se requiere promover estrategias para el desarrollo sustentable, entendiendo esto como una nueva forma de producir bienes públicos y proporcionar servicios que satisfaga las necesidades de las comunidades humanas y no humanas, mejorando la calidad de vida en general, pero en una relación de protección y respeto con el medioambiente (Zarta, 2018).

Parte medular de esta crisis planetaria se ha sostenido bajo la cultura del desarrollo económico, tecnológico y científico como forma de evolución del ser humano y que regula de manera importante la forma de convivir social y medioambientalmente, pero que ya no logra una respuesta frente al colapso climático en curso. Esta condición es precisamente lo que constituye una crisis, puesto que se requiere de un cambio de paradigma urgente, de lo que ontológicamente entendemos por medioambiente, y lo que significa convivir con/bajo este (Steffen et al., 2015).

En general, las mediciones a nivel mundial evidencian una importante disminución de la extensión y condición de los ecosistemas naturales, a tal punto que se espera en un futuro cercano una sexta extinción masiva del $75 \%$ de todas las especies del planeta. Bradshaw et al. (2021) exponen las dimensiones del ecosistema más alteradas por el ser humano a la fecha. La primera, (a) la pérdida de la biodiversidad, muestra un daño enorme con cerca de 700 vertebrados y 600 especies de plantas extintas 
durante los últimos 500 años, además de la rápida desaparición de los insectos, el deterioro de la biodiversidad marina y la disminución de los ríos y los lagos. El segundo, (b) el crecimiento de la población mundial, que actualmente se ha duplicado desde 1970 y se espera que continúe en expansión, con un impacto en la inseguridad alimentaria, los riesgos de salud, la degradación del suelo, la contaminación ambiental y los problemas sociales como el desempleo y el hacinamiento habitacional. Sumado a lo anterior, (c) el calentamiento global, que se estima en un aumento de 1.5 grados Celsius entre los años 2030 y 2052 (Intergovernmental Panel on Climate Change, 2018) y podría agravarse más aun para el año 2100, con el consecuente aumento de eventos extremos.

En América Latina, este cambio ambiental global ha generado más riesgos socionaturales, con daños a la biodiversidad, el incremento del nivel del mar, olas de calor, además de mayores afectaciones por eventos hidrometeorológicos que incluyen sequías e inundaciones (Sandoval-Diaz et al., 2021).

Frente a esta emergencia en curso, Steffen et al. (2015) proponen un modelo de interacción con el medio ambiente, a partir de los límites planetarios para el funcionamiento de ciertas variables biofísicas claves para la sustentabilidad del planeta. Sin embargo, el principal desafío de esta propuesta lo representa el comportamiento de las personas para regularse ante estos límites en un planeta que aún podría ser sustentable.

A nivel social, en muchos países del mundo y sobre todo en Latinoamérica, el esfuerzo por lograr un mayor desarrollo económico se ha asociado con graves problemáticas ligadas a la salud, la empleabilidad, la educación y la pobreza bajo un modelo económico capitalista (Estenssoro, 2015). Formighieri y Bezerra da Costa (2020) reflexionan sobre las paradojas del modelo económico actual, dada la existencia en el mundo de suficiente alimento para abastecer a toda la humanidad, no obstante que aún 800 millones de personas en el planeta siguen siendo víctimas de la hambruna. El ejemplo anterior refleja uno de los tantos efectos de la inequidad y del aumento de la vulnerabilidad estructural de los grupos más desfavorecidos (Bradshaw et al., 2021; Sandoval-Diaz et al., 2021). A lo anterior se suma un incremento en el uso de la violencia y la migración forzada para la solución de conflictos en las sociedades, con el consecuente deterioro de la salud mental (Charlson et al., 2019; Glavic, 2020; ONU, 2020). En este último punto, por ejemplo, en el continente americano se evidencia un creciente aumento de la carga de trastornos mentales, junto con el incremento de la brecha para su tratamiento (Kohn et al., 2018).

Una respuesta que cobra cada vez mayor relevancia frente a lo anterior, es que acompañado de la reducción de la vulnerabilidad estructural, debemos sumar a las respuestas institucionales preventivas frente a los riesgos, el fortalecimiento de las capacidades locales y la resiliencia comunitaria (Sandoval-Diaz et al., 2021). Esto se podría lograr desde un enfoque educativo, en donde el desafío de la sustentabilidad incluya una transformación en la formación de los profesionales como un elemento clave.

\section{La formación profesional}

Existe un acuerdo común en que la educación de calidad es fundamental para el desarrollo y crecimiento de las personas y de la sociedad en su conjunto. La formación profesional formal es aquel periodo educativo en donde las personas aprenden de manera planificada, sistemática y aplicada los saberes disciplinarios en el contexto de la educación terciaria.

Bajo un conjunto de demandas sociales e institucionales, la educación superior ha debido reformarse durante estas últimas décadas para formar profesionales que respondan a los cambios tecnológicos, económicos, sociales y del mercado. Básicamente, la difícil relación entre la formación 
universitaria y el mundo del trabajo, la compleja articulación entre teoría y práctica, además de las exigencias sociopolíticas de formar ciudadanos para el desarrollo sustentable, han tensionado a las instituciones de educación superior (Jääskelä et al., 2016). En una misma línea, Wang (2021) advierte de un contexto social actual en donde a las universidades se les exige cada vez más una adecuada vinculación con el gobierno y la industria, en tanto ciencia aplicada e innovación tecnológica. Además de demandarles la vinculación con el territorio aportando a la solución de problemas locales.

En la búsqueda de una respuesta a lo expuesto en el párrafo anterior, la Unión Europea ha hecho hincapié en formar profesionales para el crecimiento económico sustentable, la creación de mejores puestos de trabajo, además de la promoción de la competencia y la innovación en el mundo laboral, en tanto objetivos que plasmaron en el influyente acuerdo de Bolonia y han trascendido a muchos países del mundo (Jääskelä et al., 2016). Bajo un enfoque formativo basado en competencias, se espera que los profesionales logren saber pensar, saber decir, saber hacer y querer pensar, decir y hacer. Esto, bajo la lógica de formar profesionales capaces de abordar los complejos problemas sociales y laborales actuales (Pérez \& Pérez, 2013). De esta manera, un gran número de universidades forman profesionales considerando a las competencias la base de los perfiles profesionales, las mallas curriculares y de los programas de estudio (Cuadra-Martínez et al., 2018).

Sin embargo, el complejo mundo del trabajo, sumado al aumento de la matrícula en la educación terciaria, ha generado un número importante de candidatos(as) calificados(as) dispuestos(as) a asumir cualquier tipo de trabajo. Esta competitividad laboral presiona a las universidades a formar profesionales con competencias que aseguren un mayor capital cognitivo, cultural, social y psicológico, dimensiones que serían necesarias para enfrentar los desafíos que impone el mercado laboral actual (Pham, 2021).
Pero hoy se requiere ir más allá de la formación profesional en base a competencias. Formar profesionales comprometidos y sensibles ante la crisis global requiere desarrollar profesionales sensibilizados, identificados y comprometidos con esta causa. Aunque, actualmente, hay una urgencia de instalar una educación para el desarrollo sustentable y, por ejemplo, los Objetivos de Desarrollo del Milenio y la Educación para Todos reconocen la importancia de la educación superior en esta tarea (Chankseliani et al., 2021), es imperioso repensar el rol educativo de las universidades para hacer frente a las graves problemáticas socioambientales tanto globales como locales.

En este contexto, la formación profesional representa una salida prometedora, para la cual se requiere aunar esfuerzos de planeación e implementación, esto bajo la acuciante urgencia de responder ética y eficientemente ante la crisis en curso. En este desafío, la evidencia científica muestra la importancia que tiene la identidad de los sujetos en la regulación del comportamiento con el medio social y ambiental (Pérez et al., 2020), por lo que formar sistemáticamente una IP para mitigar la crisis global, podría ser una parte de la solución, constituyéndose como un proyecto de vida profesional en sintonía con el desarrollo sustentable.

\section{La formación de la identidad profesional}

Pensar la sostenibilidad social y ambiental desde la formación de la IP es una respuesta necesaria frente a la crisis global. Esto porque la IP es lo que permite otorgar sentido y valor a la profesión, implicando un conjunto de saberes que guían las decisiones profesionales y, sobre todo, el marco éticomoral que orienta al profesional para convivir social y ambientalmente.

El concepto de IP se ha abordado desde diversas disciplinas, por lo que su definición no siempre ha alcanzado acuerdo. Sin embargo, se puede entender como una construcción personal situada en un 
contexto cultural y laboral de lo que significa ser profesional. Así, la IP posee las dimensiones individual y colectiva que interactúan para desarrollar saberes, valores, prácticas, costumbres y tradiciones propias de la profesión (Cardoso et al., 2014; Heled \& Davidovitch, 2021).

En el proceso dinámico de construcción de la IP, la persona debe establecer quién es y qué hacer como profesional, lo que incluye la reflexión y la agencia en la interacción entre el sujeto y el contexto sociocultural y laboral. Como parte de este resultado, la persona define un rol profesional y social dentro de una estructura social, desarrolla una forma de vida profesional, además de la agencia y autoestima profesional (Cardoso et al., 2014). Para Jebril (2008) son cuatro las etapas de desarrollo de la IP. La primera es una fase de preocupación por el futuro laboral, que surge en la niñez y adolescencia, incluyendo la exploración vocacional. La segunda, de formación profesional, implica integrar la identidad personal con la IP durante la formación inicial. La tercera etapa, de ejercicio profesional, permite integrar la teoría con la práctica, además del conocimiento desde el contexto laboral. La última etapa se denomina post profesional, incluyendo una reinterpretación de la IP a partir de la experiencia profesional ganada en el campo laboral.

Precisamente, la formación inicial es uno de los periodos en que más se desarrolla la IP (Beijaard, 2019). Cuando los estudiantes ingresan a la educación superior, las experiencias formativas ajustan, tensionan y desafían los saberes intuitivos y expectativas que tienen de la profesión. En la medida que avanzan en su proceso formativo, el programa de estudio, las interacciones con los formadores y sus pares, además de los símbolos que se transmiten de la profesión, van impactando en lo que implica ser un profesional y en cuánto se quiere llegar a ser ese profesional. Según Barbarà-i-Molinero et al. (2017), los factores que inciden en la IP durante la formación inicial son los siguientes: (a) la experiencia social, es decir, los símbolos, las experiencias y los conocimientos que familiares y amigos transmiten al estudiante acerca de la profesión; (b) las características de los programas de estudio a partir de los saberes formales de la disciplina y la profesión transmitidos, además de aspectos como el nombre de la profesión, la extensión del programa formativo y la heterogeneidad de los estudiantes que lo cursan; (c) la experiencia con la profesión, siendo un elemento clave la posibilidad de integrar la teoría con la práctica en contextos reales; (d) la congruencia percibida con la profesión, en términos de autoeficacia profesional, valores y creencias; (e) el involucramiento y el desarrollo personal, es decir que en la medida que los estudiantes se comprometen con el aprendizaje de la profesión y sienten que crecen como personas, desarrollan más su IP.

Contar con estrategias sistemáticas para el desarrollo de la IP en la educación superior puede favorecer la construcción de una IP reflexionada, consiente y más arraigada (Cuadra-Martínez et al., 2021). Cuando los profesionales desarrollan su IP de una forma consciente, se transforman en constructores activos de una de las dimensiones más importantes de la persona, desarrollando autoeficacia, agencia, engagement y un mejor desempeño (Beijaard, 2019). Además, la IP prescribe lo que se debe hacer o no como profesional, es decir, marca los límites de la acción profesional, por lo que regula las relaciones entre el mundo profesional, la ciudadanía y, para el presente caso, el medio ambiente.

\section{Dimensiones de la identidad profesional para enfrentar la crisis global}

Dado que la IP se compone de dimensiones e incluso subidentidades (Yang et al., 2021), este trabajo propone que la formación profesional, frente a la crisis global, considere desarrollar sistemáticamente un conjunto de dimensiones que podrían ser indispensables para el ejercicio de cualquier profesión. No se trata de diluir la IP de cada profesión, sino de integrar a la IP estas dimensiones para la sustentabilidad social y ambiental. 


\section{La integración reflexiva de saberes}

Un comportamiento de protección del medioambiente, y de constructiva convivencia social, depende de un adecuado intercambio y manejo de conocimiento (Fazey et al., 2013). En el caso de los profesionales, les corresponde elaborar, implementar y evaluar acciones, programas o estrategias relacionados directa o indirectamente con las sustentabilidad social y ambiental, por lo que el conocimiento a la base de sus decisiones profesionales puede tener un impacto enorme en las acciones de mitigación y adaptación ante la crisis global.

Fleischman y Briske (2016) consideran que los profesionales poseen un conocimiento sobre lo ecológico de tipo (a) local, que se caracteriza por ser intuitivo, subjetivo y basado en la experiencia cotidiana; (b) científico, que se organiza en base a teorías científicas; y (c) profesional ecológico, que surge en el contexto laboral. Para estos autores, el conocimiento profesional ecológico no logra articularse adecuadamente con el saber científico y tampoco con el conocimiento subjetivo-local, siendo insuficiente y en ocasiones inapropiado para la sustentabilidad planetaria.

El conocimiento acerca de lo que es la profesión y de lo que implica ser un profesional es clave y se organiza como una dimensión importante de la IP. Quienes se forman como profesionales desarrollan saberes, valores, prácticas, costumbres y tradiciones propias de la profesión (Cardoso et al., 2014). Pero a menudo esto no se logra con estrategias sistemáticas de formación profesional en la educación terciaria porque las universidades no han considerado suficientemente en sus planes de estudio formar la IP.

Dado que la evidencia científica advierte de una importante relación entre el conocimiento ambiental, las actitudes y el comportamiento proambiental (Araghi et al., 2014; Jensen, 2002; Liu et al., 2015; Mark, 2011; Saribas, 2015), es necesario que el conocimiento que se tiene acerca de lo que significa ser profesional y de lo que implica como rol, incluya procesos sistemáticos de integración de estos saberes que surgen en el contexto laboral o el conocimiento profesional ecológico, el conocimiento formal que predomina en la formación universitaria y el conocimiento intuitivo o subjetivo (Fleischman \& Briske, 2016).

Frente a la crisis global, formar una IP en base al conocimiento científico es insuficiente, dado que las personas se relacionan con el medio social y ambiental desde una dimensión experiencial, desde donde construyen un conocimiento de lo cotidiano, a menudo con más sentido y significado, por lo que muchas formas de convivencia social y de relación con el medio ambiente dependen de este tipo de conocimiento de carácter situado. Por otro lado, tampoco parece adecuado que la IP solo integre un conocimiento intuitivo de lo que implica convivir social y ambientalmente. En el contexto del cuidado medioambiental, para Al-Rabaani y Al-Shuili (2020), la mayoría de los problemas son causados por el estilo de vida y las actividades de desarrollo de las personas, producto del desconocimiento y las actitudes de consumo inadecuadas sobre los impactos ambientales.

Un problema importante que enfrenta la formación profesional es lograr integrar estos saberes. A las universidades se les critica por la predominancia de una enseñanza basada en conocimiento académico (teorías científicas y no científicas), desarticuladas de los problemas locales y reales de la sociedad, lo que dificulta aplicaciones concretas y la integración de este conocimiento en la IP (Pérez \& Pérez, 2013). De esta manera, el aprendizaje en la formación profesional, es vivenciado como distante y desarticulado de la realidad, sin negar con esto lo relevante de este tipo de conocimiento para hacer frente a la crisis global.

Una forma de lograr desarrollar una IP que incluya un saber integrado por el conocimiento 
subjetivo, profesional y científico es, en primer lugar, (a) desarrollar una epistemología integradora de la formación profesional que posicionen en un mismo valor a estos tres tipos de saberes. Esto podría orientar a los programas formativos a la consideración de estrategias sistemáticas de aprendizaje de la profesión considerando el saber experiencial con el que los estudiantes interpretan el mundo, intersectando esto con las teorías científicas a la base de la profesión y con el saber práctico, contexto en el que emerge el conocimiento profesional. (b) La sistematización de la integración de estos saberes implica el uso de ciertas herramientas que la psicología cognitiva ha venido desarrollando desde hace algunas décadas, como la necesaria explicitación de creencias, en este caso sobre lo que significa ser un profesional frente a la crisis global, y la necesaria capacidad reflexiva de los estudiantes sobre este saber, su relación con la toma de decisiones profesionales y articulación con el conocimiento científico. (c) Por último, una dimensión importante de la IP es la autoeficacia profesional. La formación profesional debe incluir una reflexión permanente en el estudiantado del saber que posee, la forma en que lo ha construido y la autoeficacia profesional desarrollada a partir de este. No es suficiente con desarrollar un saber integrado como parte de la IP. También se requiere que estos sean conscientes de que lo poseen y de lo crítico de esto para hacer frente a la crisis global. Ciertamente, desarrollar un saber integrado y reflexionado como parte de la IP, puede conllevar a que los profesionales se sientan autoeficaces para explicar y accionar frente a la compleja crisis global.

\section{Creatividad para el desarrollo sustentable}

La crisis global ha generado un escenario amenazante y caracterizado por un alto dinamismo del riesgo por desastres socionaturales, además de crisis sociales por problemáticas cada vez más agudas (tales como las migraciones forzadas por el cambio climático), por lo que la incertidumbre parece ser una constante que tensiona la sustentabilidad social y ambiental.
Los profesionales de hoy necesitan forjar una IP que incluya la capacidad de adaptarse de manera rápida y eficiente frente a los dinámicos problemas y desafíos que impone la crisis global. El desarrollo científico, tecnológico y económico logrado, además de las formas actuales de convivencia social y ambiental, ya no son suficientes para hacer frente a la crisis global. Se requieren respuestas nuevas, eficientes y bien elaboradas, por lo que la creatividad en la formación profesional es clave frente a la crisis global (Orkibi, 2021).

Formar profesionales con una IP integrada por una dimensión creativa para la sustentabilidad ambiental, implica desarrollar la capacidad para identificar y definir problemas, planear e implementar respuestas originales y eficientes frente a la crisis global, en donde el significado de la profesión y su ejercicio, integra como sello distintivo el ser y sentirse un profesional con respuestas nuevas frente a los viejos/ clásicos problemas.

De hecho, la crisis medioambiental global está conllevando a que las organizaciones instalen dispositivos de gestión «verde» de recursos humanos en donde el desarrollo y fomento de la creatividad es indispensable (Ali et al., 2020). Esto es fundamental, por ejemplo, para lograr una economía ecológica, puesto que se requiere de profesionales capaces de transformar completamente el ciclo de producción y consumo (Cuadra-Martínez et al., 2017).

Además, hay evidencias de una relación entre la creatividad de las personas y el comportamiento proambiental (Corral-Verdugo et al., 2015; Fraj \& Martinez, 2006; Sandri, 2013). Algunos estudios han encontrado que el desarrollo de la creatividad de los estudiantes desarrolla emociones positivas como la felicidad, el bienestar subjetivo, la motivación y la autoconfianza (Jindal-Snape et al., 2013; Sellman, 2012), en tanto, estados psicológicos positivos que cumplirían una función mediadora del comportamiento proambiental (Cheng, 2018). 
De esta manera, el pensamiento y la resolución creativa de problemas son una necesidad frente a la complejidad, así como componentes significativos de la educación para la sustentabilidad (Cheng, 2018). La formación de una IP creativa, podría considerar (a) el significado atribuido a la profesión, puesto que es necesario que se reconozca como una característica importante de la profesión, a la creatividad; (b) el sentido de la profesión, ya que se requiere que el ejercicio de la profesión se entienda como un desempeño creativo para el desarrollo sustentable; (c) los saberes acerca de la creatividad, específicamente, los que implican ser un profesional creativo y cuáles son las estrategias para lograr un pensamiento creativo; (d) la autoeficacia creativa, ya que también es necesario que los profesionales se sientan capaces de resolver problemas socioambientales de manera elaborada, original y eficiente.

\section{Identidad profesional ecológica}

Quienes desarrollan una identidad vinculada al medio ambiente natural, presentan una mayor sensibilidad y respeto por la naturaleza, se preocupan más por los problemas ecológicos, y despliegan mayores conductas de protección hacia el medio natural (Clayton et al., 2021). Desde un enfoque social, una identidad ambientalista implica la identificación de las personas con grupos sociales cuya causa de movilización sea la protección del medio ambiente (Brick \& Lai, 2018). Desde una perspectiva psicológica, una identidad ambiental se refiere a la construcción de sentidos y significados sobre sí mismo, en una estrecha conexión con la naturaleza, junto a la creencia de autoeficacia para desplegar un repertorio de conductas de protección ambiental (Clayton et al., 2021). Por otra parte, el término identidad ecológica integra los conceptos anteriores, utilizándose para dar cuenta del desarrollo de una identidad tanto ambientalista como ambiental.

En el caso de los docentes, por ejemplo, cuando desarrollan una IP ecológica, se esfuerzan por planificar e implementar una variedad de enfoques de educación ambiental, logrando una importante conexión con sus estudiantes al compartir experiencias y valores ecológicos, y orientándose a desarrollar capacidades en los estudiantes para la construcción de un mundo más respetuoso con el medio (Rushton, 2021).

Para formar una IP ecológica, en este trabajo se sugiere que la educación universitaria debería considerar como parte esencial de sus modelos educativos las variables que la literatura ha identificado para su desarrollo. Una de estas es la conexión con la naturaleza, que incluye la forma en que se define el medio ambiente y al sujeto en relación con la naturaleza, además de los valores morales a la base de esto. Otro factor es el sentimiento de pertenencia e integración en los grupos sociales proambientales. En este enfoque social, la práctica, la acción y el reconocimiento son promotores de una identidad ambientalista, lo que implica una forma de situarse en el mundo obteniendo reconocimiento por las acciones ambientales. Lo anterior está basado en tres etapas: (a) toma de conciencia de los problemas ambientales, (b) identificación y autodefinición como un actor que contribuye al cuidado ambiental y (c) conocimiento sobre cuidado y protección del medio ambiente (Riggs, 2015).

\section{Identidad profesional prosocial}

No obstante, forjar una IP ecológica podría ser insuficiente. Planear e implementar acciones profesionales proambientales, sustentadas en un saber integrado y creativo para hacer frente a la crisis global, podría ser una fuente de alta tensión en la esfera política, económica, científica y profesional por la resistencia al cambio en las formas de convivir. Es por esto que se requiere formar profesionales dispuestos a colaborar e implicarse en el cuidado activo de la sociedad en general. La prosocialidad se define como un comportamiento positivo que consiste en proporcionar una ayuda voluntaria que permite resolver un problema o una necesidad en el benefactor, sin esperar una recompensa a cambio. Para Neaman et al. (2018), la educación ambiental 
se ha focalizado en la relación entre el ser humano y el medio natural, descuidando un elemento central como lo es las diferentes formas de convivir. Formar una IP prosocial implica que los profesionales se definan a sí mismos como tolerantes, compasivos, cuidadosos y responsables por la vida del otro, lo que podría orientar a un comportamiento profesional sustentable con acciones destinadas a proteger tanto al medio natural como al humano (Corral-Verdugo et al., 2015; Tapia et al., 2013).

Hay evidencia de una relación positiva entre el comportamiento prosocial y el comportamiento proambiental (Corral-Verdugo et al., 2015; Ruiz \& Cortes, 2021; Tapia et al., 2013) e incluso para Neaman et al. (2018) ambos tipos de comportamiento podrían integrar un constructo psicosocial mayor necesario de comprender y abordar en la educación ambiental para la sustentabilidad planetaria.

Asimismo, la atención de calidad de parte de los profesionales depende, en gran medida, de la capacidad de estos para apoyarse mutuamente en la solución de los problemas que aquejan a los clientes, además de una relación de apoyo genuino entre el profesional y el cliente. Algunos estudios han encontrado evidencia de lo anterior, por ejemplo, en profesionales de la salud (Liebe et al., 2019) y en el campo de la educación (Jennings \& Greenberg, 2009). De esta forma, frente a la crisis global, es necesario que la educación superior también se oriente a construir una IP prosocial, integrando en este proceso formativo (a) los significados que los estudiantes poseen sobre la bondad y maldad humana, (b) desarrollando valores prosociales y conocimientos sobre lo que implica ser un profesional prosocial, (c) además de instalar procesos formativos prácticos en donde puedan desplegar la ayuda a los necesitados, por ejemplo, en base a aprendizaje más servicio (Alvarado et al., 2019; Sandoval-Diaz et al., 2021).

La Figura 1 grafica la construcción de una IP considerando las dimensiones integración de saberes, creatividad, ecológica y prosocial.

\section{Figura 1}

Propuesta de formación de la IP para hacer frente a la crisis social

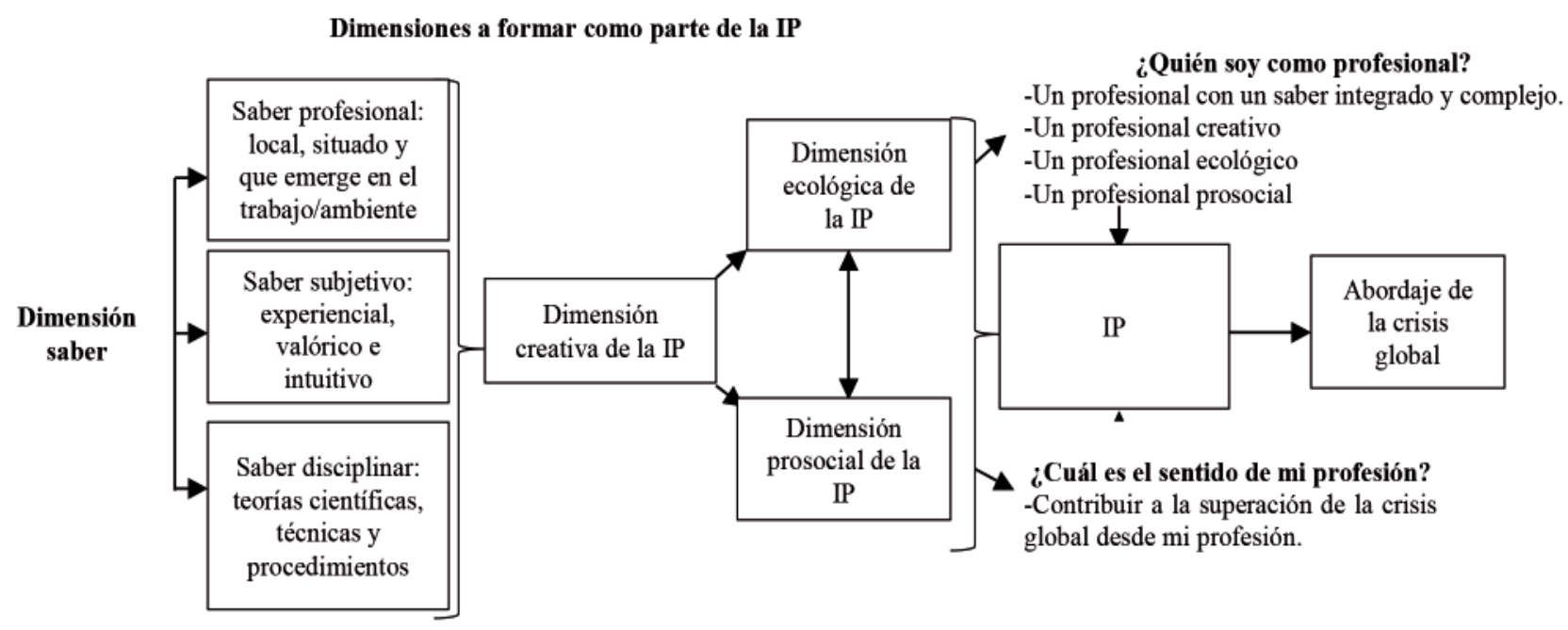




\section{Conclusiones}

La evidencia científica actual alerta de un riesgo sin precedentes para el desarrollo y mantención de los ecosistemas (Dasgupta, 2021; Glavic, 2020; Tregidga \& Laine, 2021). A pesar de que se han buscado soluciones políticas y tecnocientíficas para disminuir los riesgos y que la ciencia ha aportado conocimiento para comprender el fenómeno, la crisis global social y climática sigue en aumento (Glavic, 2020; Steffen et al., 2015; UNESCO, 2014).

En este trabajo, se propone, como una de las soluciones posibles, sumar a la respuesta gubernamental y política ante los riesgos socioambientales (Sandoval-Diaz et al., 2021), el fortalecimiento de las capacidades de uno de los actores protagónicos para el desarrollo social: los profesionales. Lo anterior, bajo un enfoque de formación de desarrollo sistemático de la IP, por ser esta una de las dimensiones más importantes en la vida de las personas (Barbarà-i-Molinero et al., 2017) teniendo la capacidad de orientar de manera importante el ejercicio profesional.

Si desde hace algunas décadas las críticas y la exigencia a la educación superior se vienen focalizando en la distancia de lo formativo con respecto al mundo del trabajo, actualmente, el desafío está puesto en formar profesionales que logren dar respuesta a la crisis global. Para ello, desarrollar sistemáticamente la IP es un camino prometedor porque es en base a esto que las personas le otorgan un sentido a la profesión y se orientan para ejercerla. Pero no se trata de desarrollar cualquier IP, este sentido de la profesión se debe alinear con la urgencia para el desarrollo sustentable frente a la crisis global (Chankseliani et al., 2021).

En este trabajo se propone formar sistemáticamente una IP para hacer frente a la crisis global en base a cuatro dimensiones. La primera dimensión, de integración de saberes, implica formar profesionales con una IP compuesta por saberes integrados reflexivamente. Lograr esta integración de saberes, así como también la transdisciplinariedad de los equipos, habilita a los profesionales y organizaciones para planear e implementar respuestas más elaboradas y eficientes frente a una compleja crisis global. Esto porque posibilita que el ejercicio profesional se oriente de una manera más congruente y reflexiva al integrar los conocimientos subjetivos, profesionales y científicos a la base de cualquier lineamiento profesional. Aunque no es una tarea sencilla para las universidades, actualmente, existen herramientas que podrían aportar a una planificación sistemática de una IP construida en base a estos tres saberes (Cuadra-Martínez et al., 2018).

Una segunda dimensión necesaria de integrar en la formación de la IP es la creatividad. Para modificar la forma de interactuar con el medio social y ambiental, es necesario pensar e implementar nuevas respuestas. Formar profesionales con un pensamiento y una autoimagen profesional creativos, implica desarrollar sensibilidad frente a los problemas sociales y ambientales, capacidad para definir y redefinir estas problemáticas, planear e implementar respuestas elaboradas e innovadoras, además de generar autoeficacia para hacer frente a la crisis global (Cheng, 2018).

Lo anterior podría resultar en un profesional altamente identificado con la profesión, lo que implica vocación, saberes necesarios para prestar servicios a la sociedad, además de un pensamiento creativo y un sentimiento de autoeficacia para ejercer la profesión. Sin embargo, se necesita que la IP desarrollada esté al servicio de la sustentabilidad social y ambiental. Para esto, se ha propuesto en este trabajo integrar a la formación de la IP las dimensiones prosocial y ambiental. Es necesario que los profesionales de hoy se identifiquen como profesionales prosociales, para que desde allí orienten el ejercicio de la profesión a la solución de las necesidades y problemáticas urgentes de la crisis global. Lograr esto favorece un ejercicio profesional cuyo norte es precisamente ayudar al prójimo. Además, la prosocialidad profesional promueve el 
apoyo entre los equipos de trabajo y la adecuada relación profesional-beneficiario (Corral-Verdugo et al., 2015; Ruiz \& Cortes, 2021; Tapia et al., 2013).

Por último, una dimensión ecológica de la IP permite que los profesionales se sientan conectados con la naturaleza, desarrollen sentimientos positivos con esta, además de valores ecológicos, para que desde allí orienten un ejercicio profesional de respeto y protección de la naturaleza (Clayton et al., 2021; Rushton, 2021).

Resta decir que repensar la formación profesional como un proceso orientado a la construcción sistemática de una IP comprometida con la crisis global, podría ser una respuesta prometedora para la sustentabilidad del planeta. Sin embargo, se requiere seguir aportando al tema, para orientar este complejo desafío de formar profesionales altamente identificados con la sustentabilidad social y ambiental.

\section{Conflicto de intereses}

Este trabajo no presenta conflictos de intereses.

\section{Responsabilidad ética}

Los autores indican que para esta investigación no se han realizado experimentos en seres humanos ni en animales, dado que corresponde a un artículo teórico.

\section{Contribución de Autoría}

DC-M: elaboración del manuscrito.

PC-C: revisión del manuscrito, escritura general y apoyo en búsqueda de bibliografía.

COV: revisión general del artículo y apoyo en la elaboración de la conclusión.

IG-P: revisión general del artículo y apoyo en la elaboración del desarrollo del artículo.

JSD: revisión general del artículo y apoyo en la elaboración de la introducción del artículo.
DPZ: revisión general del artículo y apoyo en la elaboración de la conclusión.

\section{Agradecimientos}

Este trabajo se enmarca en el proyecto Fondecyt. El equipo agradece a la Agencia Nacional de Investigación y Desarrollo ANID.

\section{Referencias}

Ali, C., Islam, A., Chung, S., Mohammad, N., \& Afrin, M. (2020). A Study of Green Human Resources Management (GHRM) and Green Creativity for Human Resources Professionals. International Journal of Business and Management Future, 4(2), 57-67. https:/ /doi.org/10.46281/ijbmf.v4i2.857

Alvarado, R., Pradenas, C., Yañez, N., Cuadra, D., \& Sandoval, J. (2019). Teorías subjetivas del comportamiento prosocial: significados, desarrollo y motivaciones de jóvenes voluntarios ante un desastre socionatural. Liberabit, 25(2), 251-266. https://doi.org/ 10.24265/liberabit.2019.v25n2.08

Al-Rabaani, A., \& Al-Shuili, A. (2020). Environmental Knowledge, Attitudes, and Behavior among Omani Post-Basic Education Students. European Journal of Social Sciences, 60(1), 29-38. https://www. europeanjournalofsocialsciences.com/issues/PDF/ EJSS_60_1_03.pdf

Araghi, Y., Kroesen, M., Molin, E., \& van Wee, B. (2014). Do Social Norms Regarding Carbon Offsetting Affect Individual Preferences towards this Policy? Results from a Stated Choice Experiment. Transportation Research, 26, 42-46. http://dx.doi.org/10.1016/ j.trd.2013.10.008

Barbarà-i-Molinero, A., Cascón-Pereira, R., \& HernándezLara, A. (2017). Professional Identity Development in Higher Education: Influencing Factors. International Journal of Educational Management, 31(2), 1-27. http:/ /dx.doi.org/10.1108/JJEM-05-2015-0058

Beijaard, D. (2019). Teacher Learning as Identity Learning: Models, Practices, and Topics. Teachers and Teaching, 25(1), 1-6. https://doi.org/10.1080/13540602.2 019.1542871 
Bradshaw, C., Ehrlich, P., Beattie, A., Ceballos, G., Crist, E., Diamond, J., Dirzo, R., Ehrlich, A., Harte, J., Harte, M., Pyke, G., Raven, P., Ripple, W., Saltré, F., Turnbull, C., Wackernagel, M., \& Blumstein, D. (2021). Underestimating the Challenges of Avoiding a Ghastly Future. Frontiers in Conservation Science, 1, 1-10. https://doi.org/10.3389/fcosc.2020.615419

Brick, C., \& Lai, C. K. (2018). Explicit (but not Implicit) Environmentalist Identity Predicts Pro-Environmental Behavior and Policy Preferences. Journal of Environmental Psychology, 58, 8-17. https://doi.org/ 10.1016/j.jenvp.2018.07.003

Cardoso, I., Batista, P., \& Graça, A. (2014). Professional Identity in Analysis: A Systematic Review of the Literature. The Open Sports Science Journal, 7(Suppl2, M2), 83-97. http://doi.org/10.2174/1875399X014070 10083

Chankseliani, M., Qoraboyev, I., \& Gimranova, D. (2021). Higher Education Contributing to Local, National, and Global Development: New Empirical and Conceptual Insights. Higher Education, 81, 109-127. https:// doi.org/10.1007/s10734-020-00565-8

Charlson, F., van Ommeren, M., Flaxman, A., Cornett, J., Whiteford, H., \& Saxena, S. (2019). New WHO Prevalence Estimates of Mental Disorders in Conflict Settings: a Systematic Review and Meta-Analysis. Lancet, 94(10194), 240-248. http://dx.doi.org/10.1016/ S0140-6736(19)30934-1

Cheng, V. (2018). Views on Creativity, Environmental Sustainability and Their Integrated Development. Creative Education, 9(5), 719-743. http://dx.doi.org/ 10.4236/ce.2018.95054

Clayton, S., Czellar, S., Nartova-Bochaver, S., Skibins, J. C., Salazar, G., Tseng, Y. C., Irkhin, B., \& Monge-Rodriguez, F. S. (2021). Cross-Cultural Validation of a Revised Environmental Identity Scale. Sustainability, 13(4), 112. https://doi.org/10.3390/su13042387

Corral-Verdugo, V., Tapia-Fonllem, C., \& Ortiz-Valdez, A. (2015). On the Relationship Between Character Strengths and Sustainable Behavior. Environment and Behavior, 47(8), 877-901. https://doi.org/10.1177/ 0013916514530718

Cuadra-Martínez, D., Véliz, D., Sandoval, J., \& Castro, P. J. (2017). Aportes a la economía ecológica: una revisión de estudios latinoamericanos sobre subjetividades medio ambientales. Psicoperspectivas. Individuo y Sociedad, 16(2), 1-14. http://doi.org/10.5027/ psicoperspectivas-vol16-issue2-fulltext-970

Cuadra-Martínez, D., Castro, P., \& Juliá, M. (2018). Tres saberes en la formación profesional por competencias: integración de teorías subjetivas, profesionales y científicas. Formación Universitaria, 11(5), 19-30. https://doi.org/10.4067/S0718-50062018000500019

Cuadra-Martínez, D., Castro-Carrasco, P., Oyanadel, C., \& González-Palta, I. (2021). Identidad profesional docente en la formación universitaria: una revisión sistemática de estudios cualitativos. Formación Universitaria, 14(4), 79-92. https://doi.org/10.4067/S0718-5006 2021000400079

Dasgupta, P. (2021). The Economics of Biodiversity: The Dasgupta Review. London, Reino Unido.

Elizalde, A., \& De la Cuadra, F. (2019). Hacia una nueva moral civilizatoria: la dimensión socio-ecológica en la propuesta del Laudato si’. Polisemia, 15(27), 82-105. http://doi.org/10.26620/uniminuto.polisemia. 15.27.2019.82-105

Estenssoro, F. (2015). El ecodesarrollo como concepto precursor del desarrollo sustentable y su influencia en América Latina. Universum, 30(1), 81-99. http:// dx.doi.org/10.4067/S0718-23762015000100006

Fazey, I., Evely, A. C., Reed, M. S., Stringer, L. C., Kruijsen, J., White, P. C. L., Newsham, A., Jin, L., Cortazzi, M., Phillipson, J., Blackstock, K., Entwistle, N., Sheate, W., Armstrong, F., Blackmore, C., Fazey, J., Ingram, J., Gregson, J., Lowe, P., Morton, S., \& Trevitt, C. (2013). Knowledge Exchange: a Review and Research Agenda for Environmental Management. Environmental Conservation, 40(1), 19-36. http://dx.doi.org/10.1017/ S037689291200029X

Fitzgerald, A. (2020). Professional Identity: A Concept Analysis. Nursing Forum, 55(3), 447-472. http:// doi.org/10.1111/nuf.12450

Fleischman, F., \& Briske, D. (2016). Professional Ecological Knowledge: An Unrecognized Knowledge Domain Within Natural Resource Management. Ecology and Society, 21(1). http://dx.doi.org/10.5751/ES-08274-210132 
Formighieri, R., \& Bezerra da Costa, I. (2020). The Pandemic Health Crisis and Its Implications for Food and Nutritional Security in Latin America. Portuguese Journal of Public Health, 38, 166-175. http://doi.org/ $10.1159 / 000512958$

Fraj, E., \& Martinez, E. (2006). Environmental Values and Lifestyles as Determining Factors of Ecological Consumer Behaviour: An Empirical Analysis. Journal of Consumer Marketing, 23(3), 113-144. https://doi.org/ 10.1108/07363760610663295

Glavic, P. (2020). Identifying Key Issues of Education for Sustainable Development. Sustainability, 12(16), 1-18. http://doi.org/10.3390/su12166500

Gorlacheva, E., \& Tikhomirova, T. (2021). A Measurement Instrument of Cognitive Economics in the BRICS Countries. BRICS Journal of Economics, 2(1), 40-52. http://doi.org/10.38050/2712-7508-2021-28

Heled, E., \& Davidovitch, N. (2021). Personal and Group Professional Identity in the $21^{\text {st }}$ Century Case Study: The School Counseling Profession. Journal of Education and Learning, 10(3), 64-82. https://doi.org/ 10.5539/jel.v10n3p64

Intergovernmental Panel on Climate Change. (2018). Summary for Policymakers. En V. Masson-Delmotte, P. Zhai, H. O. Pörtner, D. Roberts, J. Skea, P. Shukla, A. Pirani, W. Moufouma-Okia, C. Péan, R. Pidcock, S. Connors, J. Matthews, Y. Chen, X. Zhou, M. Gomis, E. Lonnoy, T. Maycock, M. Tignor, \& T. Waterfield, (eds.), Global Warming of $1.5^{\circ} \mathrm{C}$ (pp. 1-24). IPCC.

Jääskelä, P., Nykänen, S., \& Tynjälä, P. (2016): Models for the Development of Generic Skills in Finnish Higher Education. Journal of Further and Higher Education, 42(1), 2-14. http://doi.org/10.1080/ 0309877X.2016.1206858

Jebril, M. Y. (2008). The Evolution and Measurement of Professional Identity [tesis de doctorado, Universidad de la Mujer de Texas]. Repository@TWU. https:// hdl.handle.net/11274/10773

Jennings, P., \& Greenberg, M. (2009). The Prosocial Classroom: Teacher Social and Emotional Competence in Relation to Student and Classroom Outcomes. Review of Educational Research, 79(1), 491-525. http:/ /doi.org/10.3102/0034654308325693
Jensen, B. (2002). Knowledge, Action and ProEnvironmental Behaviour. Environmental Education Research, 8(3), 325-334. https://doi.org/10.1080/ 13504620220145474

Jindal-Snape, D., Davies, D., Collier, C., Howe, A., Digby, R., \& Hay, P. (2013). The Impact of Creative Learning Environments on Learners: A Systematic Literature Review. Improving Schools, 16(1), 21-31. https:// doi.org/10.1177/1365480213478461

Kohn, R., Ali, A., Puac-Polanco, V., Figueroa, C., López-Soto, V., Morgan, K., Saldivia, S., \& Vicente, B. (2018). Mental Health in the Americas: An Overview of the Treatment Gap. Revista Panamericana de Salud Pública, 42(e165), 1-10. https://doi.org/10.26633/RPSP.2018.165

Liebe, U., Naumann, E., \& Tutic, A. (2019). Prosocial Behavior Across Professional Boundaries: Experimental Evidence from Hospitals. SAGE Open, 9(2), 1-12. https:/ /doi.org/10.1177/2158244019846691

Liu, S., Yeh, S., Liang, S., Fang, W., \& Tsai, H. (2015). A National Investigation of Teachers' Environmental Literacy as a Reference for Promoting Environmental Education in Taiwan. Journal of Environmental Education, 46(2), 114-132. https://doi.org/10.1080/ 00958964.2014.999742

Mark, M. (2011). An Investigation on the Environmental Knowledge, Attitudes and Behavior of Maltese Youth. US-China Education Review, B(3), 413-422.

Moorhead, B. (2019). Transition and Adjustment to Professional Identity as a Newly Qualified Social Worker. Australian Social Work, 72(2), 1-13. https:// doi.org/10.1080/0312407X.2018.1558271

Neaman, A., Otto, S., \& Vinokur, E. (2018). Toward an Integrated Approach to Environmental and Prosocial Education. Sustainability, 10(3), 1-11. http://doi.org/ 10.3390/su10030583

Organización de las Naciones Unidas (ONU). (2020). A New Era of Conflict and Violence. https://www.un.org/en/ un75/new-era-conflict-and-violence

Organización de las Naciones Unidas (ONU). (2021a, 5 de mayo). Los conflictos y las crisis económicas empujan a la inseguridad alimentaria a su nivel más alto en cinco años. https://news.un.org/es/story/2021/05/ 1491682 
Organización de las Naciones Unidas (ONU). (2021b, 17 de agosto). Cambio climático: América Latina será una de las regiones más afectadas. https://news.un.org/es/ story/2021/08/1495582?fbclid=IwAR0C9JqgFvDv BohrMXGtkOEq3fp9e5yza5-fAsYq0rGCgUIlAI rrStTLmIo

Orkibi, H. (2021). Creative Adaptability: Conceptual Framework, Measurement, and Outcomes in Times of Crisis. Frontiers in Psychology, 11, 1-13. https:// doi.org/10.3389/fpsyg.2020.588172

Pham, T. (2021). Reconceptualising Employability of Returnees: What Really Matters and Strategic Navigating Approaches. Higher Education, 81, 13291345. https://doi.org/10.1007/s10734-020-00614-2

Pérez, A., \& Pérez, L. (2013). Competencias docentes en la era digital. La Formación del pensamiento práctico. Temas de Educación, 19(1), 67-84. https://revistas. userena.cl/index.php/teduacion/article/view/381

Pérez, R., Tapia-Fonllem, C., Fraijo-Sing, B., Nieblas, N., \& Poggio, L. (2020). Psychosocial Predispositions Towards Sustainability and Their Relationship with Environmental Identity. Sustainability, 12(17), 1-13. http://doi.org/10.3390/su12177195

Ribers, B., Miller, G., \& Revsbech, C. (2021). Education, Collaboration and Pedagogical Phronesis: Essential Dimensions in Professional Learning and Development. Professional Development in Education. https:// doi.org/10.1080/19415257.2021.1902835

Riggs, S. (2015). Environmental Identity Development Through Social Interactions, Action, and Recognition. The Journal of Environmental Education, 46(2), 94113. https://doi.org/10.1080/00958964.2014.1000813

Ruiz, C., \& Cortes, A. (2021). Sustainable Behaviors, Prosocial Behaviors, and Religiosity in Colombia. A First Empirical Assessment. Environmental Challenges, 4, 112. https://doi.org/10.1016/j.envc.2021.100088

Rushton, E. (2021). Building Teacher Identity in Environmental and Sustainability Education: The Perspectives of Preservice Secondary School Geography Teachers. Sustainability, 13(9), 5321. https://doi.org/10.3390/su13095321

Sandoval-Diaz, J., Cuadra-Martínez, D., Orellana-Fonseca, C., \& Sandoval-Obando, E. (2021). Diagnóstico comunitario ante desastres climáticos: una experiencia de aprendizaje-servicio. Alteridad, 16(1), 23-37. https:/ /doi.org/10.17163/alt.v16n1.2021.02

Sandri, O. J. (2013). Exploring the Role and Value of Creativity in Education for Sustainability. Environmental Education Research, 19(6), 765-778. https://doi.org/10.1080/13504622.2012.749978

Saribas, D. (2015). Investigating the Relationship between Preservice Teachers' Scientific Literacy, Environmental Literacy and Life-Long Learning Tendency. Science Education International, 26(1), 80-100.

Sellman, E. (2012). Creative approaches to inclusion. En E. Sellman (ed.), Creative Learning for Inclusion: Creating Learning to Meet Special Needs in the Classroom (pp. 3-14). Routledge.

Steffen, W., Richardson, K., Rockström, J., Cornell, S. E., Fetzer, I., Bennett, E. M., Biggs, R., Carpenter, S. R., de Vries, W., de Wit, C. A., Folke, C., Gerten, D., Heinke, J., Mace, G. M., Persson, L. M., Ramanathan, V., Reyers, B., \& Sörlin, S. (2015). Planetary Boundaries: Guiding Human Development on a Changing Planet. Science, 347(6223), 736-747. https://doi.org/10.1126/ science. 1259855

Tapia-Fonllem, C., Corral-Verdugo, V., Fraijo-Sing, B., \& Durón-Ramos, M. F. (2013). Assessing Sustainable Behavior and its Correlates: A Measure of ProEcological, Frugal, Altruistic and Equitable Actions. Sustainability, 5(2), 711-723. http://doi.org/10.3390/ su5020711

Tregidga, H., \& Laine, M. (2021). On Crisis and Emergency: Is it Time to Rethink Long-Term Environmental Accounting? Critical Perspectives on Accounting, 111. https://doi.org/10.1016/j.cpa.2021.102311

UNESCO. (2014). Global Action Programme on Education for Sustainable Development as FollowUp to the United Nations Decade of Education for Sustainable Development after 2014. https:// esdcenter.jp/wp-content/uploads/2016/04/GAP.pdf

Wang, Q. (2021). Higher Education Institutions and Entrepreneurship in Underserved Communities. Higher Education, 81, 1273-1291. https://doi.org/10.1007/ s10734-020-00611-5 
Yang, S., Shu, D., \& Yin, H. (2021). «Teaching, my Passion; Publishing, my Pain»: Unpacking Academics' Professional Identity Tensions Through the Lens of Emotional Resilience. Higher Education. https:// doi.org/10.1007/s10734-021-00765-w
Zarta, P. (2018). La sustentabilidad o sostenibilidad: un concepto poderoso para la humanidad. Tabula Rasa, 28, 409-423. https://doi.org/10.25058/20112742.n28.18

\section{David Cuadra-Martínez}

Académico del departamento de Psicología, Facultad de Humanidades y Educación, Universidad de Atacama, Chile.

Doctorado en Psicología, Facultad de Psicología, Universidad del Aconcagua. Mendoza, Argentina

ORCID: http://orcid.org/0000-0002-0810-2795

Autor corresponsal: david.cuadra@uda.cl

Pablo Castro-Carrasco

Académico del departamento de Psicología, Facultad de Humanidades, Universidad de La Serena, Chile.

ORCID: http://orcid.org/0000-0002-8640-5820

pablocastro@userena.cl

Cristián Oyanadel Véliz

Académico del departamento de Psicología, Universidad de Concepción, Chile.

ORCID: https://orcid.org/0000-0001-8423-6982

ps.oyanadel.cl@gmail.com

Ingrid González Palta

Académica del departamento de Psicología, Facultad de Humanidades, Universidad de La Serena, Chile.

ORCID: https://orcid.org/0000-0003-1296-4854

Igonzalez@userena.cl

José Sandoval-Díaz

Académico del departamento de Psicología, Universidad del Bio Bio. Centro de Estudios Ñuble y grupo de investigación Género, Ciudadanía y Territorio (GECIT), Universidad del Bio Bio, Chile.

ORCID: https://orcid.org/0000-0001-7247-7113

jsandoval@ubiobio.cl

Daniel Pérez-Zapata

Doctorando en Psicología, Universidad de Birmingham, facultad de Psicología, Reino Unido.

ORCID: https://orcid.org/0000-0001-9409-7170

daniel.perez.zapata@gmail.com 